\title{
Proposal of gain-matched VCSELs with a thermally actuated MEMS structure for wide temperature operations
}

\author{
Hayato Sano $^{\text {a) }}$ and Fumio Koyama \\ Precision \& Intelligence Laboratory, Tokyo Institute of Technology \\ 4259-R2-22, Nagatsuta, Midori-ku, Yokohama 226-8503, Japan \\ a)sano.h.ab@m.itech.ac.jp
}

\begin{abstract}
We propose a novel gain-matched VCSEL with a thermally actuated cantilever structure, which enables the lasing wavelength to be matched with a gain-peak wavelength even under wide temperature changes. The proposed structure includes an $\mathrm{Al}_{\mathrm{x}} \mathrm{O}_{\mathrm{y}}$ layer in an air-gap cavity, which results in the increase of the temperature dependence of lasing wavelengths. The modeling result shows a potential of wide temperature operations of over $200 \mathrm{~K}$.
\end{abstract}

Keywords: VCSEL, MEMS, optical interconnects

Classification: Optoelectronics, Lasers and quantum electronics, Ultrafast optics, Silicon photonics, Planar lightwave circuits

\section{References}

[1] Connie J. Chang-Hasnain, "Tunable VCSEL," IEEE J. Sel. Topics Quantum Electron., vol. 6, no. 6, pp. 978-987, Nov./Dec. 2000.

[2] M. Lackner, M. Schwarzott, F. Winter, B. Kogel, S. Jatta, H. Halbritter, and P. Meissnar, "CO and $\mathrm{CO} 2$ spectroscopy using a $60 \mathrm{~nm}$ broadband tunable MEMS-VCSEL at $1.55 \mu \mathrm{m}, "$ Opt. Lett., vol. 31, no. 21, pp. 31703172, Nov. 2006.

[3] T. Amano, F. Koyama, T. Hino, M. Arai, and A. Matsutani, "Design and fabrication of GaAs-GaAlAs micromachined tunable filter with thermal strain control," J. Lightw. Technol., vol. 21, no. 3, pp. 596-601, March 2003.

[4] W. Janto, K. Hasebe, N. Nishiyama, C. Caneau, T. Sakaguchi, A. Matsutani, F. Koyama, and C. E. Zah, "Athermal Operation of $1.55 \mu \mathrm{m}$ InP-based VCSEL with thermally-actuated cantilever structure," 20th International Semiconductor Laser Conference (ISLC2006), Hawaii, USA, PD1.1, pp. 121-122, Sept. 2006.

\section{Introduction}

Vertical cavity surface-emitting lasers (VCSELs) have been attracting great interest as light sources with low power consumption and small footprint. VCSELs are operated in single longitudinal mode because of their short cav- 
ity. Wide operating temperature ranges are needed for various applications of VCSELs such as optical interconnects. However, when the operating temperature range is so wide, the mismatch between a gain peak wavelength and a lasing wavelength deteriorates the laser performances, increasing the threshold and decreasing the modulation bandwidth of VCSELs. Tunable VCSELs using micro-electro mechanical systems (MEMS) structures have been studied in the past years $[1,2]$. VCSELs with a thermally actuated cantilever based on a bimorph effect enable us to control the temperature dependence of their lasing wavelengths [3]. We reported the athermal operation of $1550 \mathrm{~nm}$ VCSELs with decreasing the temperature dependence of lasing wavelengths by integrating a thermally actuated cantilever structure [4]. The temperature dependence was 50 times smaller than that of conventional single-mode lasers.

In this letter we propose a novel concept of "gain-matched" VCSELs using a thermally cantilever structure which makes the lasing wavelength to be matched with a gain-peak wavelength even under large temperature changes. We present the design of "gain-matched" VCSELs and the calculated temperature dependence of threshold currents, showing a possibility of ultra-wide operating temperature ranges. Our gain-matched MEMS VCSEL can be used for sever environments.

\section{Design and operating principle}

Figure 1 (a) shows the schematic of our proposed "gain-matched" MEMS VCSEL. The device consists of a top dielectric and GaAlAs DBR micromachined mirror, an active region and an AlGaAs bottom p-DBR including an oxide aperture, which provides optical and electrical confinement. The top MEMS mirror is a freely suspended cantilever-shaped AlGaAs n-DBR including an $\mathrm{Al}_{0.85} \mathrm{Ga}_{0.15}$ As thermal stress control layer on the top. Because of different thermal expansion coefficients in different GaAlAs compositions, we are able to obtain the thermal actuation of the cantilever for controlling the temperature dependence of resonant wavelengths. The air gap is formed by selective etching of a GaAs sacrificial layer underneath the cantilever. We newly insert an $\mathrm{Al}_{\mathrm{x}} \mathrm{O}_{\mathrm{y}}$ layer which functions as anti-reflection coating. The $\mathrm{Al}_{\mathrm{x}} \mathrm{O}_{\mathrm{y}}$ layer is formed by using the oxidation of $\mathrm{Al}_{0.98} \mathrm{Ga}_{0.02} \mathrm{As}$.

Figure 1 (b) shows the thermal actuation of a VCSEL cavity with a cantilever structure. As shown in Fig. 1 (b), the cavity includes an air-gap which is variable by a movable top MEMS mirror. A reduced thermal expansion coefficient of the top $\mathrm{Al}_{0.85} \mathrm{Ga}_{0.15}$ As thermal stress control layer makes the cantilever to deflect upward against temperature increase, which results in the cavity expansion and the red-shift of resonant wavelengths. Thus, we are able to increase the temperature dependences of lasing wavelengths by designing the cantilever structure. In the case of conventional VCSELs their temperature dependence of lasing wavelength (typically $0.07 \mathrm{~nm} / \mathrm{K}$ ) is much smaller than that of a gain-peak wavelength (typically $0.3 \mathrm{~nm} / \mathrm{K}$ for $1.1 \mu \mathrm{m}$ VCSELs). Thus large detuning among them deteriorates the modal gain with 
temperature changes as shown in Fig. 1 (c). If the lasing wavelength can be matched with a gain-peak wavelength even under temperature changes, this deterioration can be avoided. Thus, the temperature dependence is similar to that of Fabry-Perot edge emitting lasers. Various laser performances such as thresholds and modulation bandwidths can be improved in our gain-matched VCSELs.

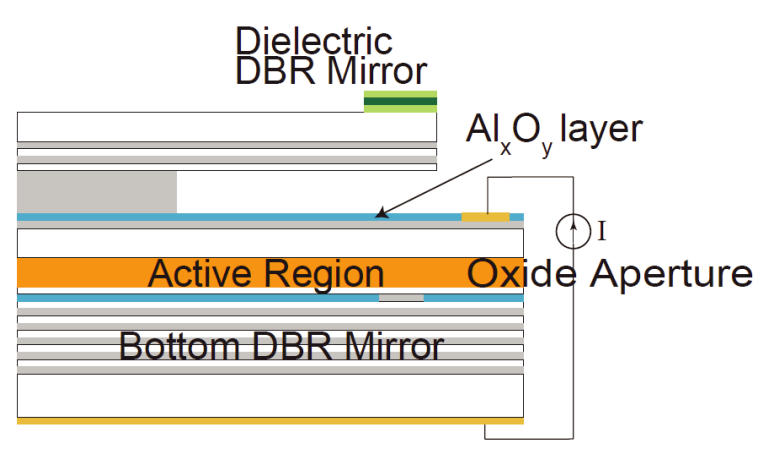

(a)

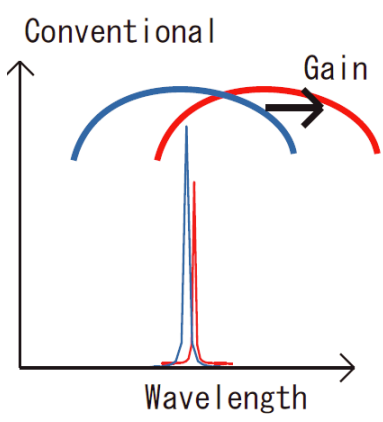

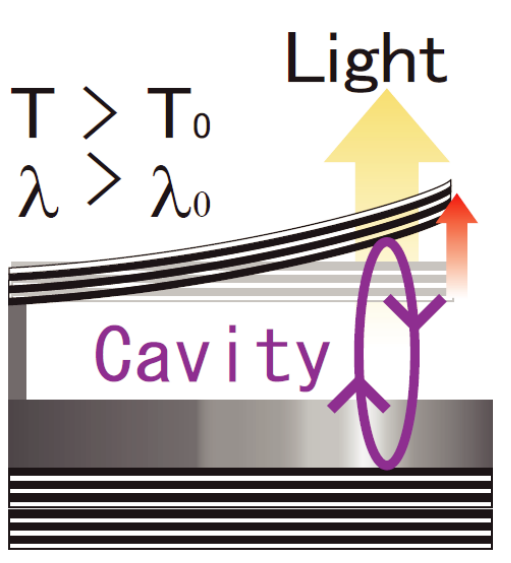

(b)

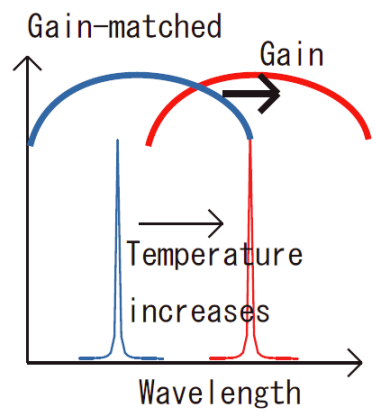

(c)

Fig. 1. (a) The schematic, (b) the thermal actuation and (c) the operating principle of our proposed gainmatched VCSELs.

\section{Enhanced temperature dependence with $\mathrm{Al}_{\mathrm{x}} \mathrm{O}_{\mathrm{y}}$ anti- reflection layer}

In the case of conventional MEMS VCSELs, the wavelength control can be realized by changing an air-gap. In order to enhance the tuning efficiency $\mathrm{d} \lambda / \mathrm{dL}$ (L: the length of the air-gap), we found that the reflection at the interface between the air-gap and the semiconductor with active region should be reduced. Our proposed device includes an $\mathrm{Al}_{\mathrm{x}} \mathrm{O}_{\mathrm{y}}$ anti-reflection layer at the interface. The refractive index of $\mathrm{Al}_{\mathrm{x}} \mathrm{O}_{\mathrm{y}}$ is about 1.5 which is close to that of a perfect anti-reflection coating. The $\mathrm{Al}_{\mathrm{x}} \mathrm{O}_{\mathrm{y}}$ layer is formed by wet-oxidation at the same time with making an oxide aperture for optical and electrical confinement. We calculated the temperature dependence of the wavelength shift of gain-matched $1.1 \mu \mathrm{m}$ VCSELs with and without the $\mathrm{Al}_{\mathrm{x}} \mathrm{O}_{\mathrm{y}}$ layer as shown in Fig. 2 (a). The cantilever consists of 4 pairs $\mathrm{Ga}_{0.80} \mathrm{Al}_{0.20} \mathrm{As} / \mathrm{Ga}_{0.15} \mathrm{Al}_{0.85} \mathrm{As}$ 


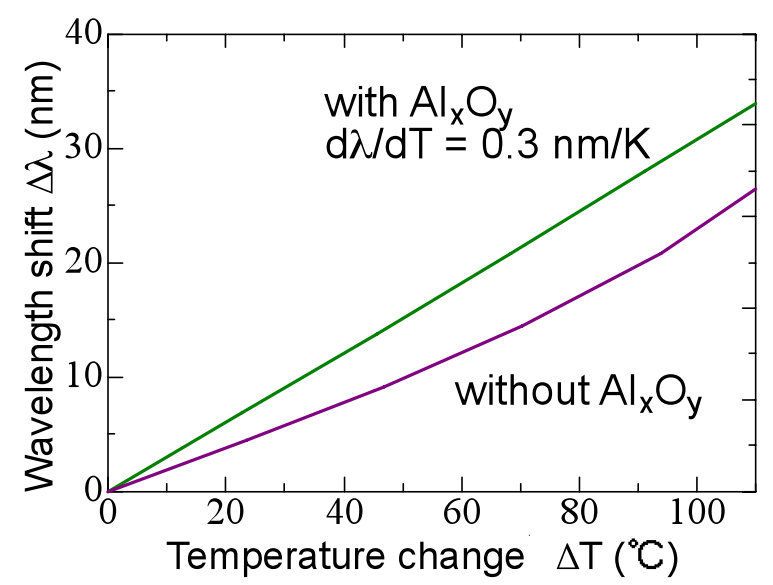

(a)

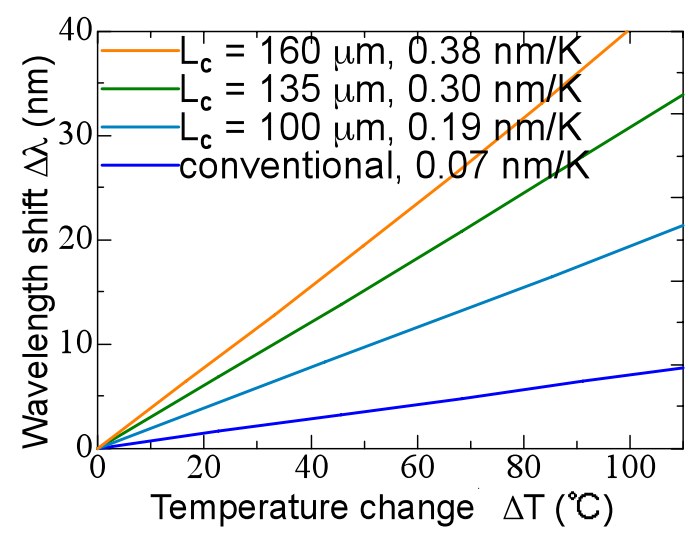

(b)

Fig. 2. (a) The calculated lasing wavelength shift versus temperature changes with and without $\mathrm{A}_{\mathrm{x}} \mathrm{O}_{\mathrm{y}}$ antireflection layer. The cantilevers is $135 \mu \mathrm{m}$ long for both structures. (b) The calculated temperature dependence of the wavelength shift for different cantilever lengths $\mathrm{L}_{\mathrm{c}}$. The wavelength shift of a conventional VCSEL without a cantilever structure is also shown.

having a $2 \lambda$-thick $(0.8 \mu \mathrm{m}) \mathrm{Ga}_{0.15} \mathrm{Al}_{0.85} \mathrm{As}$ thermal stress control layer on the top. $\mathrm{A} \lambda / 4$-thick $\mathrm{Al}_{\mathrm{x}} \mathrm{O}_{\mathrm{y}}$ anti-reflection layer is inserted for reducing the reflection at the air-gap interface. While the mechanical displacement for VCSELs with and without the $\mathrm{Al}_{\mathrm{x}} \mathrm{O}_{\mathrm{y}}$ layer against temperature changes is the same, the temperature dependence could be increased, reaching at $0.3 \mathrm{~nm} / \mathrm{K}$ for a $135 \mu \mathrm{m}$-long cantilever structure. By introducing the $\mathrm{Al}_{\mathrm{x}} \mathrm{O}_{\mathrm{y}}$ anti-reflection layer, the tuning efficiency of MEMS VCSELs could be improved, which is an advantage for our proposed structure. Figure 2 (b) shows the calculated temperature dependence of the wavelength shift for different cantilever lengths $\mathrm{L}_{\mathrm{c}}$. The wavelength shift of a conventional VCSEL without a cantilever structure is also shown for compassion. The changing rate for each line in Fig. 2 (b) shows the averaged rate while the wavelength shift is not exactly proportional to temperature changes. The temperature dependence of the lasing wavelength can be widely controlled by designing the cantilever 
structure. Based on the modeling structure with $\mathrm{L}_{\mathrm{c}}=135 \mu \mathrm{m}$, the lasing wavelength can be matched with a gain peak wavelength under large temperature changes.

\section{Temperature dependence of gain-matched VCSELs}

We calculated the temperature dependence of threshold currents as shown in Fig. 3 for our gain-matched $1.1 \mu \mathrm{m}$ VCSEL and conventional VCSEL without a cantilever structure. In the calculation we used the modal gain spectra of a strained $1.1 \mu \mathrm{m}$ triple GaAs/InGaAs quantum wells. In the modeling, we neglected excess losses such as a diffraction loss and assumed the temperature dependence of a gain-peak wavelength as $0.3 \mathrm{~nm} / \mathrm{K}$. In both cases, the lasing wavelength and the gain-peak wavelength are assumed to be matched at $60^{\circ} \mathrm{C}$. In the higher temperature region, the calculated threshold current is dominantly increased because of the decreased modal gain and the decreased reflectivity for the conventional VCSEL and the gain-matched VCSEL, respectively. However, in the lower temperature region, the threshold of the gain-matched VCSEL is dramatically improved. The result indicates a possibility of improved temperature dependences even under wide temperature range of over $200 \mathrm{~K}$.

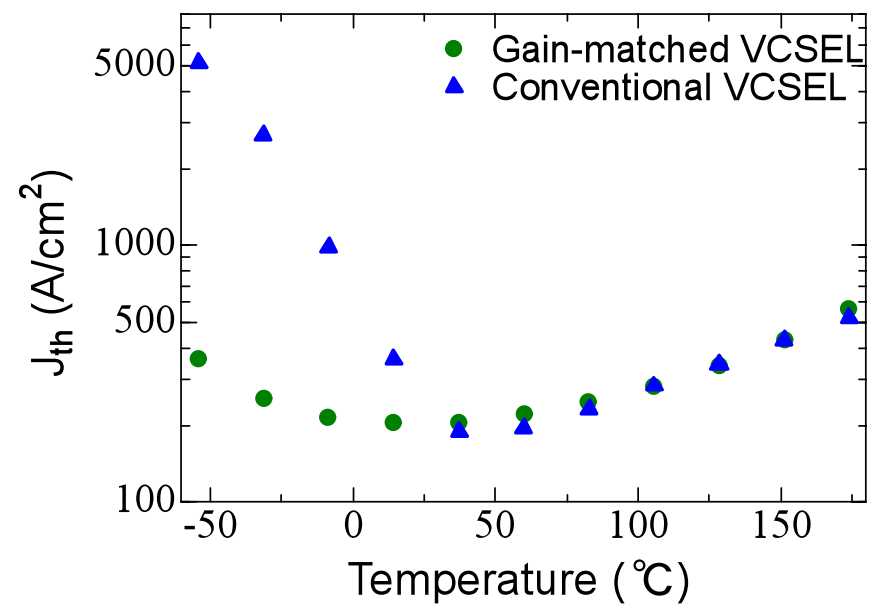

Fig. 3. The calculated temperature dependence of threshold current density for gain-matched VCSEL and conventional VCSEL without a cantilever structure.

\section{Conclusion}

We proposed a novel concept of "gain-matched" VCSELs which allow the lasing wavelength to be perfectly matched with a gain-peak wavelength even under lareg temperature changes. The proposed structure includes an $\mathrm{Al}_{\mathrm{x}} \mathrm{O}_{\mathrm{y}}$ anti-reflection layer in an air-gap cavity, which results in the increase of the temperature dependence of wavelengths. Our proposed device has a potential to be used under ultra-wide temperature range of over $200 \mathrm{~K}$. 


\section{Acknowledgments}

This work was partly supported by National Institute of Information and

Communications Technology. 